\title{
A metabolomic approach to understand the solid-state fermentation of okara using Bacillus subtilis WX-17 for enhanced nutritional profile
}

\author{
Wai Kit Mok ${ }^{1 \dagger}$, Yong Xing Tan ${ }^{2,3 \dagger}$, Jaslyn Lee ${ }^{1}$, Jaejung Kim ${ }^{1}$ and Wei Ning Chen ${ }^{1 *}$ (1)
}

\begin{abstract}
Okara is a major agro-waste produced from the soybean industry. To hydrolyze the okara and enable nutrient release, a strategy to valorize okara using solid-state fermentation with food grade Bacillus subtilis (B. subtilis) WX-17 was carried out. The study showed that fermentation of okara with B. subtilis WX-17 improved its overall nutritional content. The total amino acids content increased from $3.04 \pm 0.14 \mathrm{mg} / \mathrm{g}$ in unfermented okara to $5.41 \pm 1.21 \mathrm{mg} / \mathrm{g}$ in okara fermented with B. subtilis WX-17. Total fatty acids content increased from $153.04 \pm 5.10$ to $166.78 \pm 2.41 \mathrm{mg} / \mathrm{g}$ okara, after fermentation. Antioxidant content (DPPH) also increased by 6.4 times after fermentation. To gain insight into the mechanism, gas chromatography-mass spectrometry analysis was carried out. In total, 49 metabolites were detected, which could be classified mainly into carbohydrates, TCA cycle metabolites, amino acids and fatty acids. The decrease in carbohydrate metabolites, showed that glycolysis was upregulated. This would have provided the energy and metabolic flux towards the amino acid and fatty acid pathway. This is also in line with the increased amino acids and fatty acid production seen in okara fermented with $B$. subtilis WX-17. The findings of this work demonstrated the potential of using B. subtilis WX-17 fermentation, to enhance the nutritional profile of okara. This could serve as a potential lowcost animal feed or incorporated into the human diet.
\end{abstract}

Keywords: Bacillus subtilis, Okara, Fermentation, Metabolomics, Food waste valorisation

\section{Introduction}

Okara, also known as soy pulp is a major agro-waste produced from the soybean industry which produces soymilk and bean curd. Approximately $1.1 \mathrm{~kg}$ of okara are produced from $1 \mathrm{~kg}$ of soybean (O'Toole 1999). Okara is highly nutritious. It contains approximately $50 \%$ fiber, $25 \%$ protein, $10 \%$ lipid as well as a myriad of other high-value compounds such as isoflavones, coumestans, saponins, phytosterols, lignins and phytates. These compounds have been shown to exhibit numerous

\footnotetext{
*Correspondence: wnchen@ntu.edu.sg

†Wai Kit Mok and Yong Xing Tan contributed equally to this work

1 School of Chemical and Biomedical Engineering, Nanyang

Technological University, 62 Nanyang Drive, N1.2-B1-35,

Singapore 637459, Singapore

Full list of author information is available at the end of the article
}

physiological and therapeutic functions such as the prevention of cardiovascular diseases (CVD) in humans. However, a large amount of okara are being disposed of in landfills and incineration plants annually due to their unpalatable and insoluble nature ( $\mathrm{Li}$ et al. 2012b). It is estimated that around 14 million tonnes of okara are generated worldwide annually. The countries include Japan, Korea, China and Singapore, which contribute 800,000 tonnes, 310,000 tonnes, 2.8 million tonnes and 11,000 tonnes, respectively (Li et al. 2012b; Seong et al. 2015).

To utilize the highly nutritive compounds in the okara, pre-treatment is required to release the nutrients from the insoluble okara. Previous studies have shown that enzymes secreted by microorganisms during fermentation can hydrolyse complex macromolecules such as fatty acids, proteins and fibres into smaller and more soluble 
nutrients. It also reduces the amount of antinutritional factors present in okara including trypsin, phytic acid, lectin and tannin (Paredes-Lopez and Harry 1989). Various microorganisms such as Aspergillus sp., Aspergillus niger and Aspergillus ficuum had been studied for their ability to produce phytases which inhibited the antinutritional factor phytate. This had been shown to reduce the bioavailability of calcium, zinc and iron (Pandey et al. 2001; Schlemmer et al. 2009).

Solid state fermentation (SSF) is the culture of microorganism using solid substrate in the absence of liquid to produce desirable products. SSF has shown to be effective in enhancing the nutritional content of a complex substrate. This is because microorganism secretes abundant enzymes which catabolizes complex macromolecules into simpler forms. For example, a recent study conducted by Dessie et al. showed that fruit and vegetable wastes that underwent solid state fermentation using Aspergillus niger and Rhizopus oryzae increased in succinic acid which is an important metabolite in the tricarboxylic acid (TCA) cycle and have numerous health benefits such as antioxidant properties and strengthening of the immune system (Dessie et al. 2018; Saif and Fumio Hashinaga 2005). Bacteria, yeast and fungi are commonly used in SSF. Rhizopus, Lactobacillus, Streptococcus, Aspergillus and Bacillus are some of the most common microorganism used in solid state fermentation of food material (Hesseltine 1987).

In recent years, SSF have received increasing amount of attention from researchers and industrial players since several studies performed on colourings, flavourings, additives and other desirable products for the food industry had shown that SSF can achieve higher yield compared to SLF (Rodriguez-Couto and Sanromán 2006). It is considerably cheaper and more environmentally friendly as compared to the more commonly used submerged liquid fermentation (SLF). Some of the advantages of SSF over SLF include reduced probability of contamination due to lack of moisture and simple media composition since most nutrients are in the solid substrate. SSF also allows the use of simple reactor design due to the concentrated nature of solid substrate (Mienda and Idi 2011).

Bacillus subtilis (B. subtilis) is a microorganism of interest for fermentation of okara, due to its ability to secrete enzymes, which can break down the macromolecules in okara, as well as the ability to increase antioxidant activity. The objective of this study was to investigate the effects of SSF on okara using a strain of food grade B. subtilis WX-17, which was isolated in this study, from Natto. Then, an untargeted metabolomic approach using gas chromatography-mass spectrophotometry (GC-MS) was carried out to analyse the value-added products produced in okara fermented with B. subtilis WX-17. The mapping of metabolites unto the metabolomic pathways would also provide an important insight into the mechanisms behind solid-state fermentation of okara with B. subtilis WX-17. Till date, based on our knowledge, there exist a gap in the utilization of bacteria in valorising okara from a metabolomic perspective. The findings of this study could open up the possibility of using fermented okara as a low-cost animal feed or even supplement the human diet which could go a long way towards alleviating the global food security issue.

\section{Materials and methods \\ Chemicals}

Glycerol, nutrient broth, methanol, ribitol, methoxamine hydrochloride, N-methyl-N-(trimethylsilyl) trifluoroacetamide (MSTFA), trimethylchlorosilane (TMCS), sodium chloride, acetic acid, heptadecanoic acid, ethanol, chloroform, BF3-methanol, hexane, $\gamma$-aminobutyric acid, dimethylformamide (DMF), 1-1,-diphenyl-2-picrylhydrazil (DPPH).

\section{Microorganism}

Bacillus subtilis WX-17 was isolated from Marumiya Kyushu Ichiban Natto. This strain has been deposited in NCIMB with the accession number NCIMB 15204. The isolation was carried out by adding $20 \mathrm{~mL}$ of sterile water to 3 natto beans in a falcon tube and vortexed for $5 \mathrm{~min}$ to extract the microorganism. The cell suspension was serial diluted and plated onto nutrient agar plates and incubated at $37{ }^{\circ} \mathrm{C}$ for $24 \mathrm{~h}$. A single colony was inoculated into $5 \mathrm{~mL}$ of nutrient broth and incubated at $37{ }^{\circ} \mathrm{C}$ for $24 \mathrm{~h}$ and subsequently stored in aliquots containing $50 \%$ glycerol at $-80^{\circ} \mathrm{C}$.

\section{Bacterium identification}

A single colony was inoculated in $5 \mathrm{~mL}$ of nutrient broth and incubated overnight. The bacterial DNA were then isolated using Bio Basic EZ-10 Spin Column Fungal Genomic DNA Mini-Prep Kit. Next, PCR was carried out to amplify the $16 \mathrm{~S}$ rDNA gene of the bacteria using the forward and reverser primer 27F ( $5^{\prime}$ AGA GTT TGA TCM TGG CTC AG $3^{\prime}$ ) and 1492R (5' GGT TAC CTT GTT ACG ACT T $3^{\prime}$ ), respectively. PCR was performed with the following parameters: 35 cycles at $98{ }^{\circ} \mathrm{C} 10 \mathrm{~s}$ for denaturation, $55{ }^{\circ} \mathrm{C} 5 \mathrm{~s}$ for annealing, $72{ }^{\circ} \mathrm{C} 2 \mathrm{~min}$ for elongation and $68{ }^{\circ} \mathrm{C} 10 \mathrm{~min}$ for extension followed by cooling to $4{ }^{\circ} \mathrm{C}$. Gel electrophoresis was then carried out on the PCR sample to remove other contaminants and the DNA purified using QIAquick Gel Extraction Kit (250) before sequencing. 16s rRNA sequencing (Genbank accession number MK559744) was outsourced to Bio Basic Asia Pacific Pte Ltd using Sanger dideoxy sequencing technology. The obtained 16s rRNA sequence 
(GenBank accession number MK559744) was then compared with other 16s rRNA sequences using the BLAST algorithm (https://blast.ncbi.nlm.nih.gov/Blast.cgi).

\section{Source of okara}

Fresh okara samples were kindly provided by Vitasoy International Singapore Pte Ltd, Singapore. Okara were separated into aliquots and sealed in air tight polyethylene bags and stored at $-20^{\circ} \mathrm{C}$ in the dark.

\section{Fermentation}

Bacillus subtilis WX-17 was inoculated into $5 \mathrm{~mL}$ of nutrient broth and incubated at $37{ }^{\circ} \mathrm{C}$ for $24 \mathrm{~h}$ which served as the stock culture. $10 \mathrm{~g}$ of okara was inoculated with B. subtilis WX-17 at a concentration of $10^{6} \mathrm{CFU} / \mathrm{g}$ of okara in a petri dish. The petri dish was then covered with 2 layers of cling film. The first layer was pressed onto the inoculated okara and the second layer was wrapped across the surface of the petri dish. Both layers were punctured with numerous holes using a sterile pin to maintain aeration and moisture content within the petri dish and subsequently fermentation was carried out at $37{ }^{\circ} \mathrm{C}$ for $72 \mathrm{~h}$. A beaker of water was placed in the incubator to maintain the moisture content. The fermented okara were then freeze-dried and stored at $-20{ }^{\circ} \mathrm{C}$ until further analysis.

\section{Sample preparation for metabolomic, fatty and amino acids analyses}

For the metabolomic analysis, $3 \mathrm{~mL}$ of methanol were added to $900 \mathrm{mg}$ of fermented okara and raw okara (control), respectively. The samples were then homogenized using Fastprep- $24^{\mathrm{TM}} 5 \mathrm{G}$ Homogenizer. Homogenizing was carried out for $30 \mathrm{~s}$ at $5 \mathrm{~min}$ interval for 5 times. The tubes were placed in a box of ice after each homogenizing cycle to cool the samples. Next, the samples were centrifuged at $9000 \mathrm{~g}$ for $10 \mathrm{~min}$ at $4{ }^{\circ} \mathrm{C}$. The supernatant was extracted and filtered through a $0.22 \mu \mathrm{m}$ filter. $10 \mu \mathrm{L}$ of ribitol $(2 \mathrm{mg} / \mathrm{mL})$ were added into $1.5 \mathrm{~mL}$ of filtered supernatant as the internal standard (IS). Samples were then vortexed for $30 \mathrm{~s}$ and allowed to dry in a heat block at $30{ }^{\circ} \mathrm{C}$, overnight. Then, $100 \mu \mathrm{L}$ of methoxamine hydrochloride $(20 \mathrm{mg} / \mathrm{mL}$ pyridine $)$ was added to the lyophilized samples for methoximation to protect the carbonyls and incubated at $37^{\circ} \mathrm{C}$ for $60 \mathrm{~min}$. Next, silylation was carried out by adding $200 \mu \mathrm{L}$ of $N$-methyl$N$-(trimethylsilyl) trifluoroacetamide (MSTFA) with $1 \%$ trimethylchlorosilane (TMCS) and subsequently incubated at $70{ }^{\circ} \mathrm{C}$ for $30 \mathrm{~min}$. The samples were then centrifuged for $30 \mathrm{~min}$ at $15,330 \mathrm{~g}$ and $150 \mu \mathrm{L}$ of supernatant were transferred to glass vials and sent for GC-MS analysis.
For fatty acids analysis, $10 \mathrm{mg}$ of fermented okara and $10 \mathrm{mg}$ of fresh okara (control) were weighed and placed into eppendorf tubes. $1000 \mu \mathrm{L}$ of $0.9 \% \mathrm{NaCl}$ solution and $200 \mu \mathrm{L}$ of acetic acid were then added. $10 \mu \mathrm{L}$ of $10 \mathrm{mg} /$ $\mathrm{mL}$ heptadecanoic acid dissolved in ethanol was added to the extraction solvent to serve as IS. The solvents were then homogenized as described above. Then, $3 \mathrm{~mL}$ of a chloroform-methanol 2:1 mixture was added, and the samples were inverted several times, vortexed vigorously for $5 \mathrm{~min}$, and centrifuged at $10,000 \mathrm{~g}$ for $10 \mathrm{~min}$ at $4{ }^{\circ} \mathrm{C}$. The chloroform layer (bottom, $1 \mathrm{~mL}$ ) was collected and dried overnight at $30{ }^{\circ} \mathrm{C}$. The dried lipid residue was redissolved in $500 \mu \mathrm{L}$ BF3-methanol 10\% (FLUKA, 15716) and incubated in a sealed vial in a $95{ }^{\circ} \mathrm{C}$ heater for $20 \mathrm{~min}$. FAMEs were extracted with $300 \mu \mathrm{L} n$-hexane after the addition of $300 \mu \mathrm{L}$ saturated $\mathrm{NaCl}$ in water. Samples were vortexed for $5 \mathrm{~min}$ and centrifuged at $14,800 \mathrm{rpm}$ for $5 \mathrm{~min} .150 \mu \mathrm{L}$ of sample (top layer) was transferred into glass vials for GC-MS analysis.

For amino acids analysis, $4 \mathrm{mg}$ of fermented okara and $4 \mathrm{mg}$ of raw okara (control) were resuspended in $200 \mu \mathrm{L}$ of $6 \mathrm{M} \mathrm{HCl} .20 \mu \mathrm{L}$ of $\gamma$-aminobutyric acid $(10 \mathrm{mg} / \mathrm{mL})$ were added as IS. The tubes were sealed and baked for $24 \mathrm{~h}$ in an oven at $105^{\circ} \mathrm{C}$. The cell hydrolysate was dried at $95^{\circ} \mathrm{C}$ in a heat block. After drying, $20 \mu \mathrm{L}$ of DMF and $20 \mu \mathrm{L}$ of MSTFA were added. The tubes were sealed and incubated at $85^{\circ} \mathrm{C}$ for $1 \mathrm{~h}$. Samples were then centrifuged at 14,800 rpm for $5 \mathrm{~min}$ and supernatant were transferred to glass vials. $40 \mu \mathrm{L}$ of DMF were added into the glass vials and the vials were inverted a few times before sending for GC-MS analysis.

\section{GC-MS method for metabolomic, fatty and amino acids analyses}

Metabolomic analysis including carbohydrates and TCA cycle metabolites were carried out via GC-MS. The GCMS system (Agilent Technologies 7890A-5975C) was equipped with a HP-5MS, 5\% Phenyl-Methyl-Silox capillary column $(30 \mathrm{~m} \times 0.250 \mathrm{~mm}$ id.; $0.25 \mu \mathrm{m}$ film thickness; Agilent J\&W Scientific, Folsom, CA, USA). $1 \mu \mathrm{L}$ of samples were injected into the system by the autosampler in splitless mode. The injector temperature and ion source temperature were set at $250^{\circ} \mathrm{C}$ and $230{ }^{\circ} \mathrm{C}$, respectively. The oven temperature was as follows: $75{ }^{\circ} \mathrm{C}$ for 4 min, ramped to $280^{\circ} \mathrm{C}$ at the rate of $4{ }^{\circ} \mathrm{C} / \mathrm{min}$, and held at $280^{\circ} \mathrm{C}$ for $2 \mathrm{~min}$. Data were acquired in full scan mode from 35 to $600 \mathrm{~m} / \mathrm{z}$ with a $0.3 \mathrm{~s}$ of scan time. Metabolites were identified using the NIST08 mass spectral library based on mass spectral similarity. Samples were normalized with respect to the IS, ribitol, before comparison.

For fatty acid analysis, the injector temperature and ion source temperature were set at $250^{\circ} \mathrm{C}$ and $230^{\circ} \mathrm{C}$, respectively. The oven temperature was as follows: $80{ }^{\circ} \mathrm{C}$ for 
$1 \mathrm{~min}$, ramped to $250{ }^{\circ} \mathrm{C}$ at the rate of $7{ }^{\circ} \mathrm{C} / \mathrm{min}$, and held at $250{ }^{\circ} \mathrm{C}$ for $8 \mathrm{~min}$. Data were acquired in full scan mode from 50 to $600 \mathrm{~m} / \mathrm{z}$ at 2.66 scans per second. Metabolites were identified using the NIST08 mass spectral library based on mass spectral similarity. Samples were normalized with respect to the IS, heptadecanoic acid before comparison.

For amino acid analysis, solvent delay was set at $2 \mathrm{~min}$ $30 \mathrm{~s}$. The injector temperature and ion source temperature were set at $250{ }^{\circ} \mathrm{C}$ and $230{ }^{\circ} \mathrm{C}$, respectively. The oven temperature was as follows: $160{ }^{\circ} \mathrm{C}$ for $1 \mathrm{~min}$, ramped to $290{ }^{\circ} \mathrm{C}$ at the rate of $20{ }^{\circ} \mathrm{C} / \mathrm{min}$, ramped again to 310 at $20{ }^{\circ} \mathrm{C} / \mathrm{min}$ and held at $310{ }^{\circ} \mathrm{C}$ for $1 \mathrm{~min}$. Data were acquired in full scan mode from 180 to $550 \mathrm{~m} / \mathrm{z}$ at 3.85 scans per second. Metabolites were identified using the NIST08 mass spectral library based on mass spectral similarity. Samples were normalized with respect to the IS, $\gamma$-aminobutyric acid before comparison.

\section{Antioxidants analysis}

$300 \mu \mathrm{L}$ of ethanol was added to $100 \mathrm{mg}$ of sample and homogenized as described above. The samples were then centrifuged at 10,000 rpm for $5 \mathrm{~min} .150 \mu \mathrm{L}$ of the supernatant were transferred to new tubes and added with $100 \mu \mathrm{L}$ of 1,1,-diphenyl-2-picryl-hydrazil (DPPH) solution and $250 \mu \mathrm{L}$ of ethanol. The samples were then incubated in a dark place for $30 \mathrm{~min}$ at room temperature. The absorbance of the mixture was measured at $515 \mathrm{~nm}$ with ethanol as blank using Nanodrop 2000c Spectrophotometer. The activities of the samples were evaluated with respect to trolox equivalent- \% signal inhibition calibration curve whereby \% signal inhibition is defined as: $\%$ Signal Inhibition $=\left(1-\frac{A_{s}}{A_{o}}\right) \times 100$.

$A_{s}$ is defined as the absorbance of the samples and $A_{o}$ is defined as the absorbance of pure DPPH.

\section{Statistical analysis}

All experiments were conducted in triplicates. Statistical analysis was carried out using MetaboAnalyst 4.0 (Xia et al. 2011, 2012; Xia and Wishart 2016). Data scaling was carried out using mean-centering and divided by the standard deviation of each variable prior to partial least squares discriminant analysis (PLS-DA) and heatmap analysis. The heatmap was also constructed using Euclidean distance measurement and ward clustering algorithm.

\section{Nucleotide sequence accession number}

The 16s rRNA sequence of WX-17 was deposited in the GenBank database with the accession number MK559744.

\section{Results}

Metabolic profiles of fermented and unfermented okara

A metabolomics analysis using GC-MS provided an overview on the metabolic profiles between the fermented and unfermented okara. Statistical analysis (PLSDA and heatmap) was carried out to understand the changes between the samples. These changes observed in the level of each metabolite, helped to shed light on the effects of $B$. subtilis WX-17 fermentation on okara. In total, 49 metabolites were detected. Figure 1 showed the PLS-DA score plot of the fermented and unfermented okara. The green and red highlights denoted the $95 \%$ confidence region. The first principal component accounted for $76.5 \%$ of the total variance while the second principal component accounted for $5.3 \%$ of the total variance which combined to explain a total of $81.8 \%$ of the variance. This showed that the first component largely explained most of the variance between the samples. From the PLS-DA score plot $\left(\mathrm{R}^{2}=99.8 \%\right.$ and $\mathrm{Q}^{2}=98.3 \%$ ), clear and distinct separations between the unfermented okara and the fermented okara along the first principal component were observed. This showed that during fermentation, the metabolic profile of okara had changed significantly.

Although the PLS-DA score plot provided a visual representation of the difference in the metabolic profile between fermented and unfermented okara, it did not provide details about the specific metabolites. Hence a clustering heatmap was constructed to provide a more

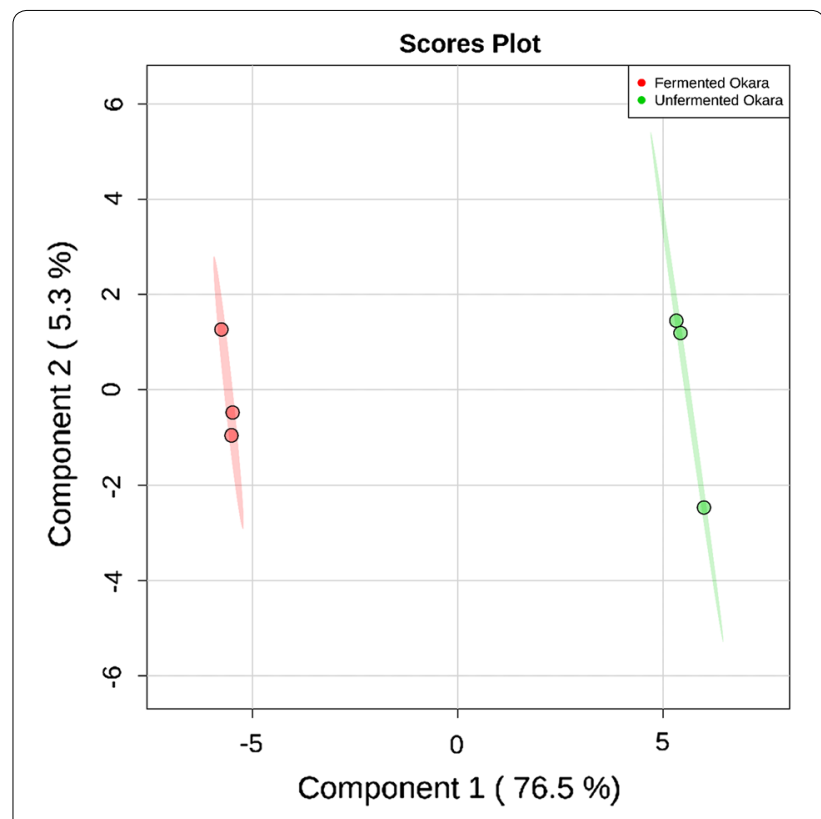

Fig. 1 PLS-DA score plot of all metabolites found for fermented and unfermented okara. The green and red highlights denoted the 95\% confidence region. Explained variance are shown in brackets 
visual breakdown of the metabolites that changed after fermentation (Fig. 2). From the heatmap, unfermented okara had high amount of carbohydrates such as fructose, ribose, glucose, galactose, mannose and maltose. In comparison, the levels of carbohydrates were lower in fermented okara. In addition, fermented okara had higher amounts of amino and fatty acids as compared to unfermented okara. This suggested that B. subtilis WX-17 consumed the carbohydrates in okara for its growth and might have produced proteases and lipases to break down proteins and lipids in okara, into simpler amino acids and fatty acids (Lesuisse et al. 1993; Yang et al. 2000).

\section{Carbohydrates and TCA cycle metabolites}

To better illustrate the effects of fermentation on fermented and unfermented okara, the results for carbohydrate metabolites and metabolites involved in the TCA

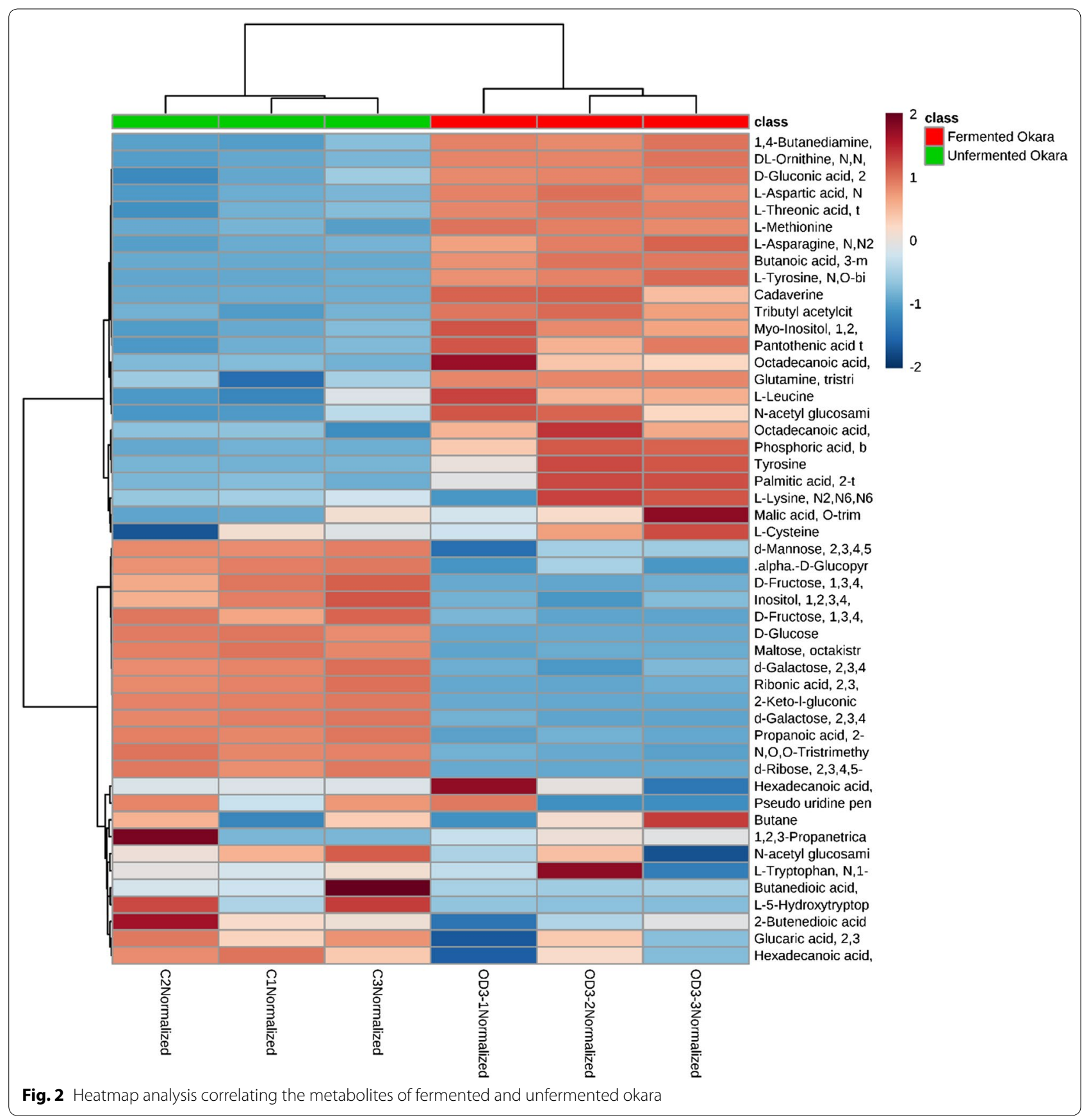


cycle were presented in Figs. 3 and 4 respectively. In total, 7 types of carbohydrates were detected. They were ribose, fructose, mannose, galactose, glucose, sucrose, and maltose. This result agreed with previous findings by Li et al. and Hou et al. which showed that the polysaccharides in soybeans and okara were ribose, galactose, glucose, fructose, sucrose, mannose and other minor components such as verbascose, pinitol and myo-inositol (Hou et al. 2009; Li et al. 2012a). The abundance of all the different types of carbohydrates were reduced after fermentation (Fig. 3).

From the metabolomics analysis, it was shown that isocitric acid and malic acid increased while succinic acid and fumaric acid decreased (Fig. 4). Most notably, isocitric acid increased by approximately 3 times. Recent studies have postulated on the potential benefits of isocitric acid. For example, Omar et al. suggested that isocitric acid contains antioxidant properties that can help to combat oxidative stress of the brain and liver in mice by decreasing the brain lipid peroxidation and inflammation, liver damage as well as DNA fragmentation (Abdel-Salam et al. 2014). Another study suggested that isocitric acid can help in combating hypoxia or hypoxic conditions such as fatigue, dizziness to more serious conditions such as hypercapnia and organ failure as it is the only metabolite in the TCA cycle that can unblock succinate dehydrogenate which would promote cell respiration even under stressful environment (Kamzolova et al. 2018).

\section{Amino acids and fatty acids metabolites}

From the heat map, it was shown that amino acids and fatty acids levels increased after fermentation. To reaffirm these findings, the amount of amino acids and fatty acids before and after fermentation were determined using GCMS protocols which were specific for amino and fatty acids. These specific protocols are more sensitive towards their respective target metabolites. Table 1 showed the absolute value of amino acids (mg/g okara) in fermented and unfermented okara. The results in Table 1 showed that all amino acids increased after fermentation with the total amount increasing by almost two-fold after fermentation. Notably, the essential amino acids leucine, phenylalanine and glutamic acid increased the most at 2.26, 2.42 and 2.12 times, respectively.

Fatty acids specific analysis suggested that there was negligible change in stearic and palmitic acids levels. However, linoleic and oleic acids levels were shown to increase by 2.93 and 2.37 times, respectively (Table 2).

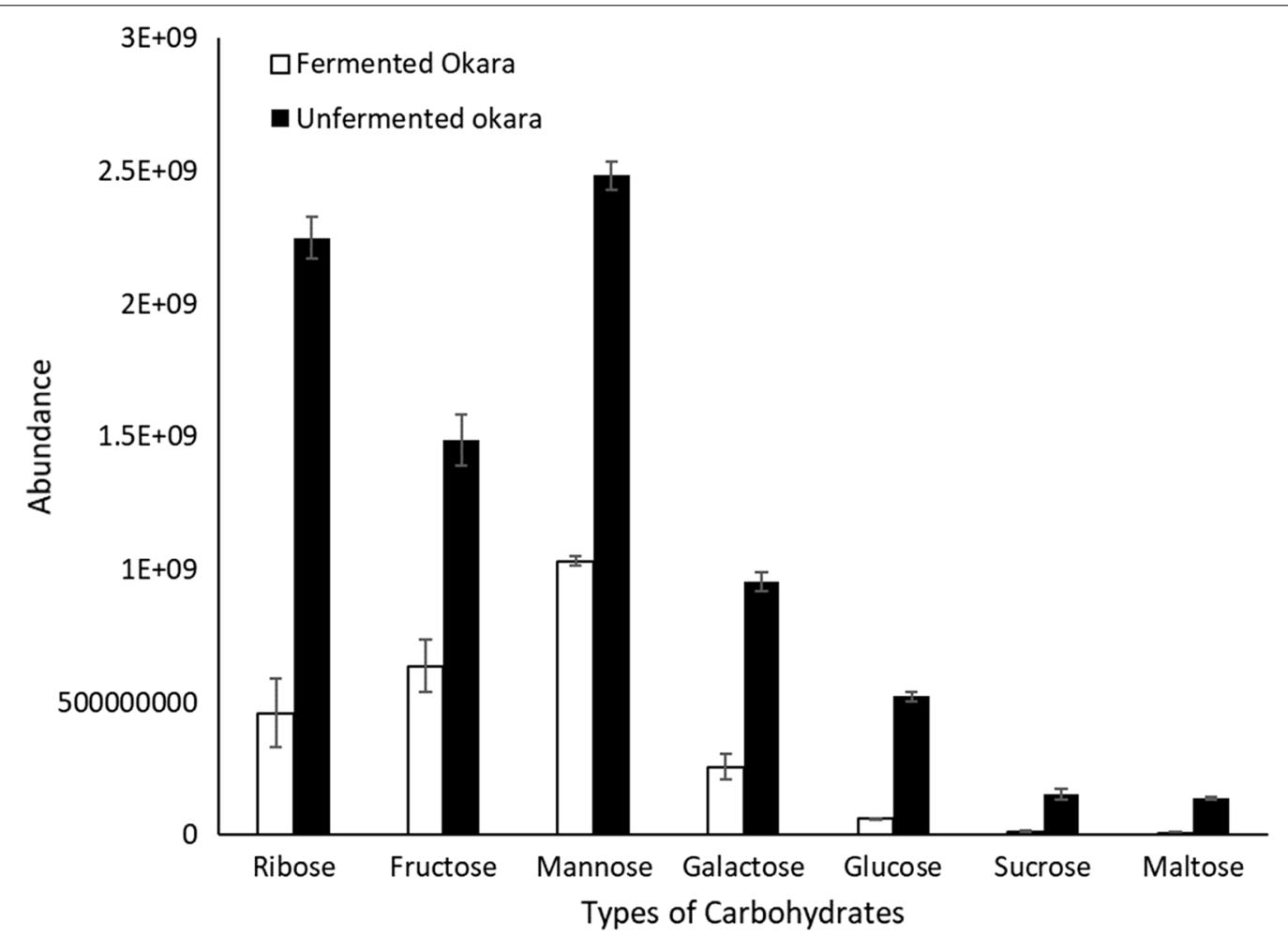

Fig. 3 Abundance of all the carbohydrates detected during analysis 


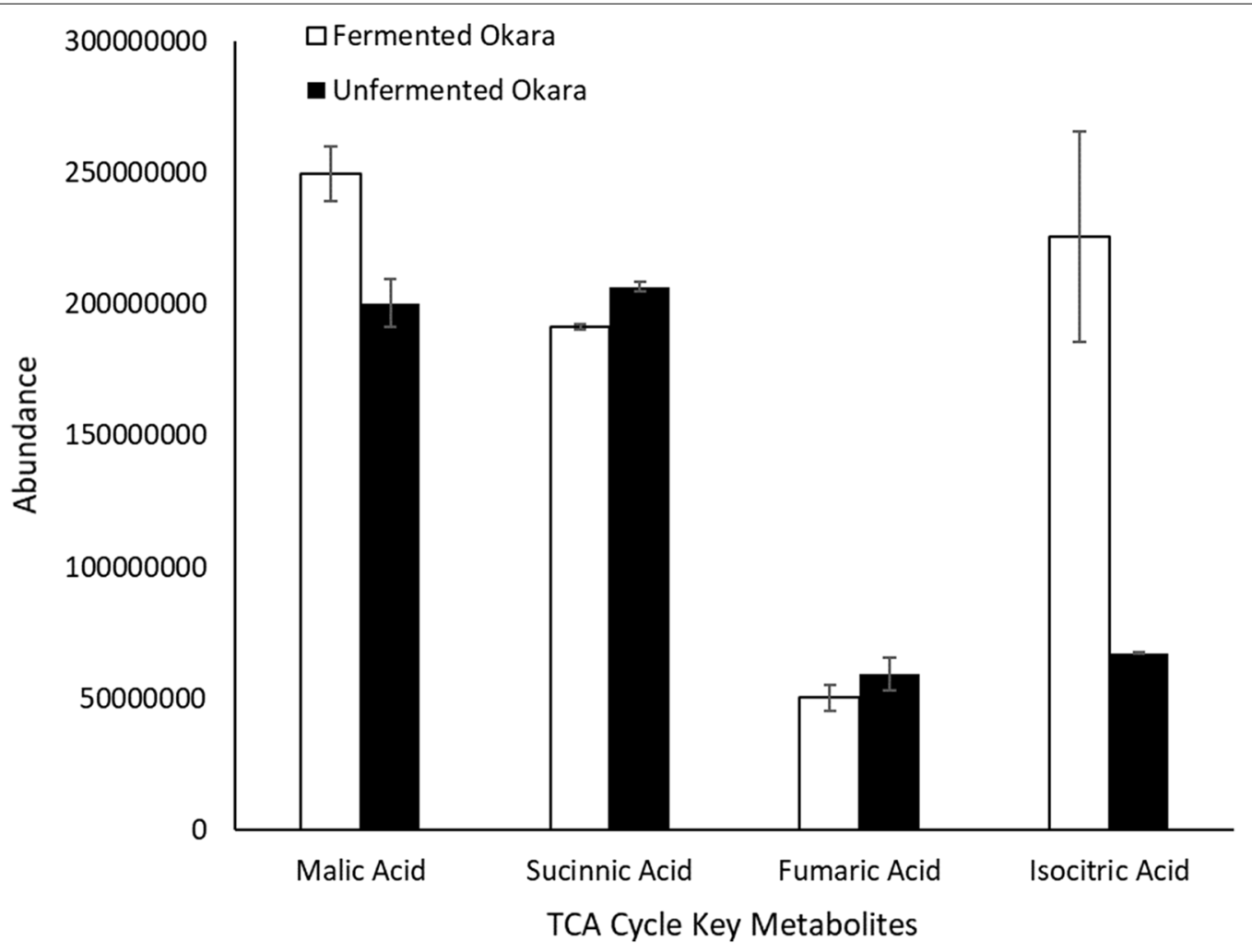

Fig. 4 Abundance of all the TCA cycle key metabolites detected

Table 1 Changes in amino acids in absolute value $(\mathrm{mg} / \mathrm{g}$ dried okara) for fermented and unfermented okara

\begin{tabular}{lcc}
\hline mg/g okara & Control (raw okara) & Fermented okara \\
\hline Glycine & $0.183 \pm 0.0441$ & $0.329 \pm 0.104$ \\
Valine & $0.0228 \pm 0.00291$ & $0.0458 \pm 0.00457$ \\
Proline & $1.28 \pm 0.442$ & $2.15 \pm 0.591$ \\
Leucine & $0.303 \pm 0.0684$ & $0.685 \pm 0.175$ \\
Serine & $0.130 \pm 0.0309$ & $0.141 \pm 0.0188$ \\
Threonine & $0.138 \pm 0.0391$ & $0.151 \pm 0.00157$ \\
Phenylalanine & $0.0799 \pm 0.0306$ & $0.194 \pm 0.0179$ \\
Aspartic acid & $0.200 \pm 0.0703$ & $0.292 \pm 0.0278$ \\
Glutamic acid & $0.611 \pm 0.0211$ & $1.30 \pm 0.182$ \\
Lysine & $0.0694 \pm 0.00989$ & $0.0856 \pm 0.0156$ \\
Tyrosine & $0.0235 \pm 0.00215$ & $0.0439 \pm 0.00352$ \\
Total amino acids & $3.04 \pm 0.136$ & $5.41 \pm 1.21$ \\
\hline
\end{tabular}

Results are as mean \pm standard deviation (3 replicates)

\section{Antioxidant activity}

The DPPH radical scavenging activity of fermented okara was shown to have increased during fermentation (Fig. 5). Fermented okara showed the highest DPPH radical scavenging activity at $43.68 \mu \mathrm{g}$ Trolox equivalent/g dried okara after $72 \mathrm{~h}$ of fermentation. As compared to the DPPH radical scavenging activity of unfermented okara at $6.79 \mu \mathrm{g}$ Trolox equivalent/g dried okara, fermented okara displayed an increased in DPPH radical scavenging activity by approximately 6.4 times.

\section{Discussion}

Metabolomics analysis showed that the overall metabolite profile of okara changed after B. subtilis WX-17 fermentation (Figs. 1 and 2). Most of the carbohydrate metabolites decreased after fermentation. This indicated that it was being consumed by B. subtilis WX-17 which could utilize the carbohydrates through glycolysis, to produce increased amounts of the energy molecule acetyl-CoA. This precursor could then enter the amino acids and fatty acid pathway. This suggested that the microorganism was able to utilise the carbon source present within okara and use it for metabolism to produce other components. It had been suggested that glucose is the preferred carbon source for B. subtilis (Singh et al. 2008; Tian et al. 2015). In this study, analysis of the various sugar pathways suggested that most other forms of carbohydrate were converted into glucose before being used for other processes.

The increase in amino acids were confirmed as shown in Table 1 . This could be due to B. subtilis producing 
Table 2 Changes in fatty acids in absolute value $(\mathrm{mg} / \mathrm{g}$ dried okara) for fermented and unfermented okara

\begin{tabular}{lcc}
\hline $\mathbf{m g} / \mathbf{g}$ okara & Control (raw okara) & Fermented okara \\
\hline Stearic acid & $60.3 \pm 2.00$ & $55.0 \pm 5.24$ \\
Oleic acid & $3.39 \pm 1.02$ & $8.04 \pm 2.87$ \\
Linoleic acid & $9.61 \pm 3.31$ & $28.2 \pm 9.55$ \\
Palmitic acid & $79.8 \pm 1.93$ & $75.6 \pm 5.37$ \\
Total fatty acids & $153.04 \pm 5.09$ & $166.78 \pm 2.41$ \\
\hline
\end{tabular}

Results are as mean \pm standard deviation (3 replicates)

extracellular proteases which would breakdown the proteins in okara into amino acids thereby contributing to their increase after fermentation. The increase in amino acids is important as they can provide as a rich source of nitrogen that are essential to living organisms. For example, yeast such as Rhodosporidium toruloides and Saccharomyces cerevisiae requires nitrogen for the synthesis of amino acids, proteins, DNA and RNA (Cruz et al. 2002; Evans et al. 1984).

Fatty acid level also increased after okara fermentation (Table 2). Particularly, the increase in both linoleic acid and oleic acid levels, were desirable as studies have reported various health benefits when these fatty acids were consumed. Linoleic acid is a polyunsaturated fatty acid that contains numerous purported health benefits such as anti-obesity, anti-carcinogenesis, anti-atherosclerosis, anti-diabetic, osteosynthetic and immunomodulation effects (Benjamin and Spener 2009; Nagao and Yanagita 2005). Likewise, oleic acid is a monounsaturated fatty acid that had been linked to a reduction in coronary heart disease due to its ability to reduce LDL-cholesterol, thrombogenicity, LDL-oxidative susceptibility as well as insulin sensitivity factors (Lopez-Huertas 2010). The increase in linoleic acid and oleic acid were expected as B. subtilis are known to produce lipases that catalyses the hydrolysis of fatty acids (Ma et al. 2006; Sánchez et al. 2002).

Endogenous metabolic processes or exogenous chemicals in food system may generate free radicals which may cause oxidative damages by oxidizing biomolecules resulting in tissue damage or even cell death. Numerous traditional fermented food such as miso, tempeh, sufu and douche have free radical scavenging ability (Zhu et al. 2008). Therefore, it is of interest to analyse if $B$. subtilis WX-17 fermented okara displayed the same ability. In this regard, analysis showed that antioxidant activity increased by 6.4 times which strengthened the case of fermented okara as a functional food for animals.

To better understand the metabolic flux during fermentation, a metabolic pathway analysis was performed that would allow a hypothetical insight into the various metabolic pathways which were up regulated or down regulated during fermentation (Fig. 6). The pathway analyses were performed with reference to the Kyoto Encyclopaedia of Genes and Genomes (KEGG) database (Kanehisa et al. 2017; Kanehisa and Goto 2000; Kanehisa et al. 2016; Ogata et al. 1999).

The precursor for glycolysis is glucose. It is converted into pyruvate through the intermediate glucose 6-phosphate (G6P) and phosphoenolpyruvate (PEP). The analysis showed that apart from ribose, all other forms of

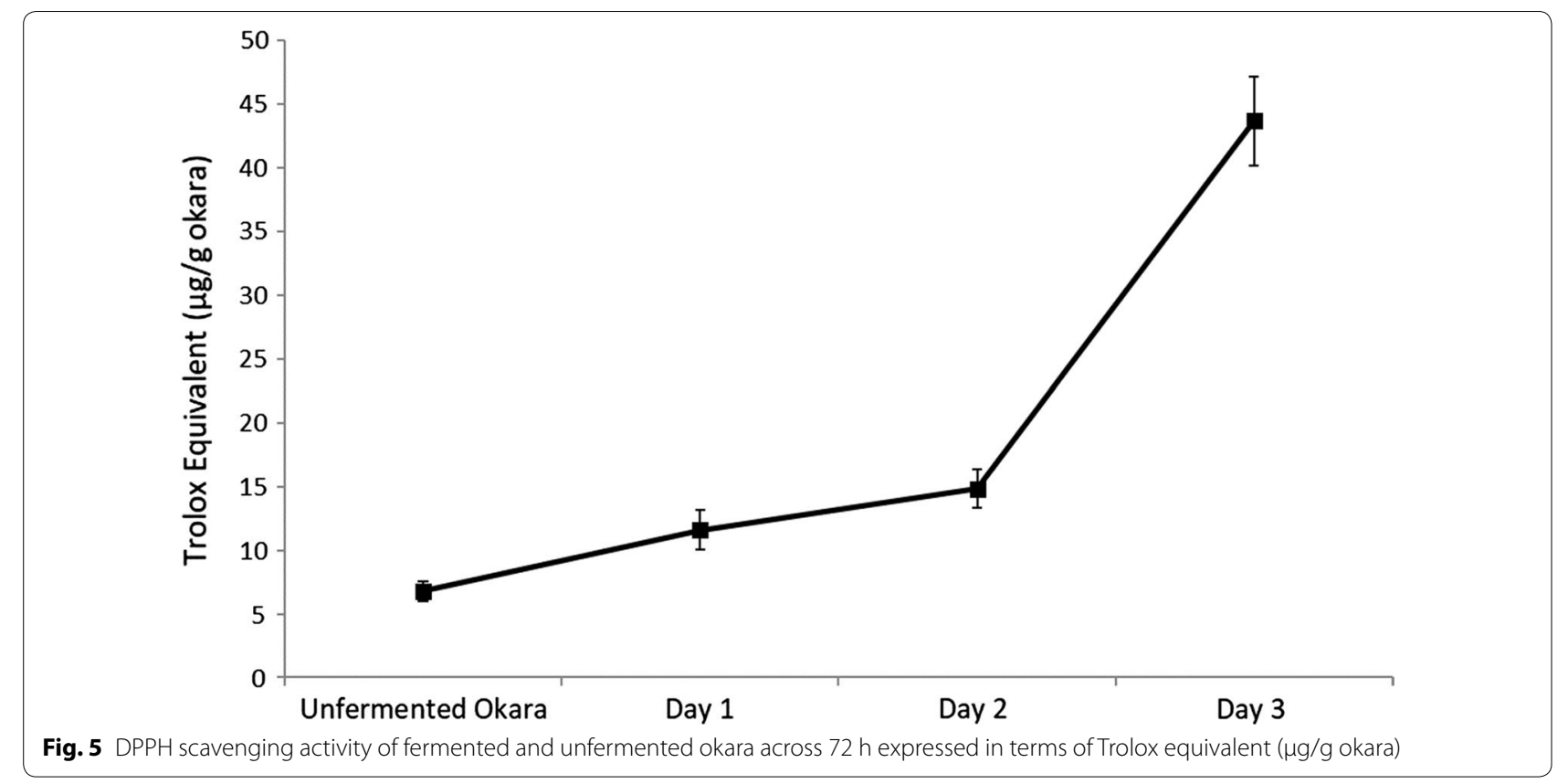




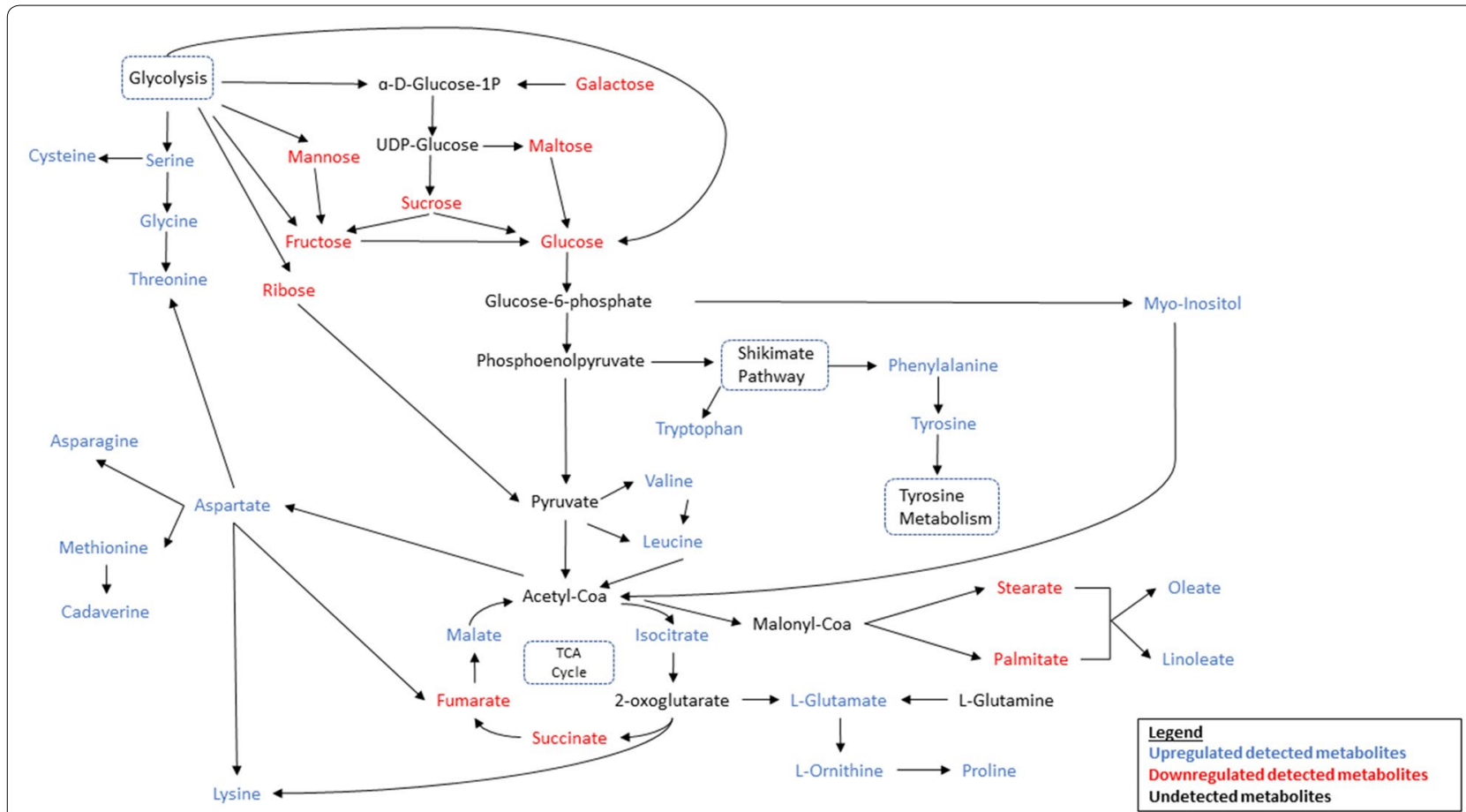

Fig. 6 Metabolic pathway analysis of all detected carbohydrates, amino acids and fatty acids after fermentation of okara

carbohydrates can be converted into glucose. Mannose can be converted into fructose through the enzyme mannose isomerase in a reversible reaction. Fructose is in turn converted into glucose via xylose isomerase. Both mannose and fructose were also produced through glycolysis through the intermediates mannan and D-fructose-2-phosphate respectively. The overall decrease in both mannose and fructose suggested that the rate of consumption is greater than the rate of production.

Glycolysis also produced sucrose through the intermediate UDP-glucose and sucrose synthase. Sucrose can then be converted into fructose and glucose through sucrose alpha-glucosidase. UDP-glucose can also be converted to maltose via maltodextrin which were then converted to glucose through maltose phosphorylase. Galactose can be converted into UDP-glucose via UDP glucose pyrophosphorylase.

The KEGG database suggested no direct pathway for conversion of ribose to glucose. This suggested that the reduction in ribose after fermentation is due to direct consumption of ribose by $B$. subtilis WX-17. This was supported by studies which showed that $B$. subtilis exhibited increased sporulation when provided with a mixture of glucose and ribose as carbon source compared to a medium with glucose as the sole carbon source (Warriner and Waites 1999).
One of the main intermediates of glycolysis, G6P can be converted into myo-inositol, a carboxylic sugar through myo-inositol 1-phosphatase (inositol phosphate metabolism pathway). During fermentation, B. subtilis WX-17 releases phytase which hydrolyses phytic acid, an antinutrient present in okara to produce myo-inositol which might explain the increased amount of myo-inositol detected after fermentation (Chen et al. 2015; Kerovuo et al. 1998). Myo-inositol can then be converted into acetyl-coA through malonate-semialdehyde dehydrogenase which can then enter the TCA cycle (inositol phosphate metabolism pathway).

Another intermediate in glycolysis, phosphoenolpyruvate (PEP) was involved in the shikimate pathway that produce tryptophan through a reversible reaction with (3-indole)-glycerol phosphate. The increased level of tryptophan detected suggested that the forward reaction (3-indole-glycerol phosphate to tryptophan) occurred at a higher rate than the backward reaction. Both phenylalanine and tyrosine are involved in the shikimate pathway as well and can be interconverted through prephenate which is an intermediate in the shikimate pathway. Their increased levels after fermentation suggested that reactions along the pathway are skewed towards phenylalanine and tyrosine metabolism rather than quinate.

Both valine and leucine which are essential amino acids were produced from the glycolysis intermediate, 
pyruvate (valine, leucine, isoleucine biosynthesis pathway). Valine is produced from the intermediate, 2-oxoisovalerate which can also be irreversibly converted into leucine. In addition, pyruvate can also be converted into leucine through pyruvate metabolism. Increased levels of valine and leucine suggested that their rate of synthesis is greater than their rate of degradation as leucine can be broken down into acetyl-coA. This implied that the bulk of the acetyl-coA were converted from pyruvate rather than leucine.

TCA cycle is a chain of reactions that are used by aerobic organisms to release stored energy in acetyl-coA through oxidation into adenosine triphosphate (ATP) and carbon dioxide. 4 of the key components in the TCA cycle were detected, of these both isocitrate and malate increased while fumarate and succinate decreased. Results also showed that isocitrate increased the most (3 times) after fermentation. This is in line with studies that had shown that $B$. subtilis produced the enzyme aconitate hydratase which catalysed the stereo-specific isomerisation of citrate to isocitrate (Cox and Hanson 1968; Dingman et al. 1987). The large amount of isocitrate produced likely drove reactions forward to produce succinate and fumarate which are consumed to produce malate.

The intermediates of the TCA cycle are involved in numerous reactions that produced important compounds. Isocitrate are broken down by the action of isocitrate dehydrogenase into 2-oxoglutarate which can then be converted into the non-essential amino acid, glutamate. Glutamate are in turn converted into ornithine via acetylornithine deacetylase which is part of the urea cycle. Critically, ornithine cyclodeaminase catalyses the conversion of ornithine to proline which is an essential amino acid (arginine and proline metabolism pathway). Glutamate, ornithine and proline were all upregulated after fermentation which strengthens the hypothesis that isocitrate were produced in excess. 2-oxoglutarate can also be converted into lysine through the lysine biosynthesis pathway.

From the pathway analysis, aspartate played a vital role in the synthesis of numerous important compounds. Firstly, in the lysine biosynthesis pathway, aspartate is converted into lysine through catalysis by diaminopimelate decarboxylase. Aspartate can also be directly converted into the TCA cycle intermediate, fumarate by aspartase (alanine, aspartate and glutamate metabolism pathway). Next, aspartate is also directly converted into asparagine, a non-essential amino acid by asparagine synthetase (alanine, aspartate and glutamate metabolism pathway). Aspartate is also involved in the synthesis of the essential amino acid, methionine which had been linked with optimising the immune function of the human intestines (cysteine and methionine metabolism
pathway)(Ruth and Field 2013). Lastly, aspartate can be converted to threonine through threonine synthase (glycine, threonine and serine metabolism pathway). Threonine is also produced from the reversible reaction of glycine through catalysis by threonine aldolase (glycine, serine, threonine metabolism pathway). Glycine, another essential amino acid is in turn produced from the reversible reaction of serine through catalysis by glycine hydroxymethyltransferase (glycine, serine, threonine metabolism pathway). In addition, serine can also be converted to cysteine through cysteine synthase (cysteine and methionine metabolism pathway).

It was also observed that all the amino acids detected were glucogenic amino acids which means that they can be converted into glucose through gluconeogenesis. This could explain why although all the amino acids were up regulated after fermentation, the overall amount detected after fermentation were much lesser compared to fatty acids.

The metabolic pathways for the biosynthesis of fatty acids involved the conversion of acetyl-coA to malonylcoA through acetyl-coA carboxylase which were then converted to the saturated fatty acids, palmitate and stearate through the fatty acid biosynthesis pathway. Palmitate and stearate then underwent elongation and unsaturation process to yield oleate and linoleate (biosynthesis of unsaturated fatty acids pathway). Both palmitate and stearate were down regulated while oleate and linoleate were up regulated. This suggested that a larger proportion of the saturated fatty acids were converted into unsaturated fatty acids. The reduction in saturated fatty acids are desirable as it is well known that consumption of high amount of saturated fatty acids are associated with increased risk of coronary heart diseases (Zong et al. 2016).

Overall, from the metabolic pathway analysis (Fig. 6), it can be summarized that the amount of various carbohydrates in okara decreased. This suggested that carbohydrates were consumed and utilised by $B$. subtilis WX-17 through glycolysis, to produce energy for its metabolism and cellular process. Subsequently, this led to the increased antioxidant amount, amino acids and fatty acids in fermented okara.

In conclusion, with the world's population predicted to reach 9 billion by 2050, food security is becoming a rising global issue (Godfray et al. 2010). One strategy to combat these issues is the biovalorisation of food waste. With the growing popularity of soy-based products, the amount of okara produced are increasing rapidly, approximately 14 million tonnes globally every year. This work showed the possibility of reintroducing okara, a food waste back into the food chain through biovalorisation by fermentation. As the microorganism used is food grade, this study also 
presents the potential application of fermented okara into human diets in various forms. The study revealed that fermentation of okara using food grade B. subtilis WX-17 enhanced its nutrient profile. GC-MS and metabolic pathway analysis showed that both amino acids and fatty acids production increased after fermentation due to the release of hydrolases by B. subtilis WX-17 to break down complex macromolecules into simpler molecules which are easier to digest. Furthermore, antioxidant analysis also showed that post fermentation, fermented okara displayed higher antioxidant activities which indicated a higher phenolic content. Future work will include characterising the enzymes produced by $B$. subtilis WX-17, such as nattokinase, as well as other important components such as the valuable vitamin K2 MK7. In addition, downstream processing to extract the bioactive compounds from fermented okara will be carried out.

\begin{abstract}
Abbreviations
B. subtilis: Bacillus subtilis; GC-MS: gas chromatography-mass spectrometry: TCA cycle: tricarboxylic acid cycle; DPPH: 1-1,-diphenyl-2-picryl-hydrazil; CVD: cardiovascular disease; SSF: solid state fermentation; SLF: submerged liquid fermentation; MSTFA: N-methyl-N-(trimethylsilyl) trifluoroacetamide; TMCS: trimethylchlorosilane; DMF: dimethylformamide; DNA: deoxyribonucleic acid; PCR: polymerase chain reaction; IS: internal standard; BF3-methanol: boron trifluoride methanol; PLS-DA: partial least squares discriminant analysis; RNA: ribonucleic acid; LDL: low density lipoprotein; KEGG: Kyoto Encyclopaedia of Genes and Genomes; G6P: glucose 6-phosphate; PEP: phosphoenolpyruvate; UDP: uridine diphosphate; ATP: adenosine triphosphate.
\end{abstract}

\section{Authors' contributions}

WNC conceived the motivation behind the project. WKM and YXT designed and performed the experiments as well as analysed the data. JK provided technical advice on experimental set-up and data analysis. WKM and YXT wrote the manuscript. JL, JK and WNC revised the manuscript. All authors read and approved the final manuscript.

\section{Author details}

${ }^{1}$ School of Chemical and Biomedical Engineering, Nanyang Technological University, 62 Nanyang Drive, N1.2-B1-35, Singapore 637459, Singapore. ${ }^{2}$ Interdisciplinary Graduate School, Nanyang Technological University, 50 Nanyang Avenue, Singapore 639798, Singapore. ${ }^{3}$ Advanced Environmental Biotechnology Centre, Nanyang Environment and Water Research Institute, Nanyang Technological University, 1 CleanTech Loop, 1 CleanTech One \#06-08, Singapore 637141, Singapore.

\section{Acknowledgements}

We would like to thank Bio Basic Singapore for technical assistance in DNA sequencing, Vitasoy International Singapore Pte Ltd for providing okara and also Dr. Xiao Mei Lyu for technical assistance and support.

\section{Competing interests}

The authors declare that they have no competing interests.

\section{Availability of data and materials}

All the data analysed in this study are included in this article. The 16s rRNA sequence of WX-17 was deposited in the GenBank database with the accession number MK559744.

\section{Consent for publication}

Not applicable.

Ethics approval and consent to participate

Not applicable.

\section{Funding}

The funding of this research was supported by the School of Chemical and Biomedical Engineering (SCBE) at Nanyang Technological University (NTU) (Tier1).

\section{Publisher's Note}

Springer Nature remains neutral with regard to jurisdictional claims in published maps and institutional affiliations.

Received: 27 March 2019 Accepted: 25 April 2019

Published online: 04 May 2019

\section{References}

Abdel-Salam OME, Youness E, Mohamed N, Morsy SMY, Omara EA, Sleem AA (2014) Citric acid effects on brain and liver oxidative stress in lipopolysaccharide-treated mice. J Med Food 17(5):588-598. https://doi.org/10.1089/ jmf.2013.0065

Benjamin S, Spener F (2009) Conjugated linoleic acids as functional food: an insight into their health benefits. Nutr Metab 6(1):36. https://doi. org/10.1186/1743-7075-6-36

Chen W, Ye L, Guo F, Lv Y, Yu H (2015) Enhanced activity of an alkaline phytase from Bacillus subtilis 168 in acidic and neutral environments by directed evolution. Biochem Eng J 98:137-143. https://doi.org/10.1016/j. bej.2015.02.021

Cox DP, Hanson RS (1968) Catabolite repression of aconitate hydratase in Bacillus subtilis. BBA Gen Subj 158(1):36-44. https://doi.org/10.1016/03044165(68)90069-X

Cruz SHD, Cilli EM, Ernandes JR (2002) Structural complexity of the nitrogen source and influence on yeast growth and fermentation. J Inst Brewing 108(1):54-61. https://doi.org/10.1002/j.2050-0416.2002.tb00124.x

Dessie W, Zhang W, Xin F, Dong W, Zhang M, Ma J, Jiang M (2018) Succinic acid production from fruit and vegetable wastes hydrolyzed by on-site enzyme mixtures through solid state fermentation. Bioresour Technol 247:1177-1180. https://doi.org/10.1016/j.biortech.2017.08.171

Dingman DW, Rosenkrantz MS, Sonenshein AL (1987) Relationship between aconitase gene expression and sporulation in Bacillus subtilis. J Bacteriol 169(7):3068. https://doi.org/10.1128/jb.169.7.3068-3075.1987

Evans CT, Ratledge C (1984) Effect of nitrogen source on lipid accumulation in oleaginous yeasts. Microbiology 130(7):1693-1704. https://doi. org/10.1099/00221287-130-7-1693

Godfray HCJ, Beddington JR, Crute IR, Haddad L, Lawrence D, Muir JF, Pretty J, Robinson S, Thomas SM, Toulmin C (2010) Food security: the challenge of feeding 9 Billion people. Science 327(5967):812. https://doi.org/10.1126/ science. 1185383

Hesseltine CW (1987) Solid state fermentation—an overview. Int Biodeterior Biodegradation 23(2):79-89. https://doi.org/10.1016/0265-3036(87)90030-3

Hou A, Chen P, Shi A, Zhang B, Wang YJ (2009) Sugar variation in soybean seed assessed with a rapid extraction and quantification method. Int J Agron 2009:8. https://doi.org/10.1155/2009/484571

Kamzolova S, Shamin RV, Stepanova NN, Morgunov GI, Lunina JN, Allayarov RK, Samoylenko V, Morgunov I (2018) Fermentation conditions and media optimization for isocitric acid production from ethanol by Yarrowia lipolytica. Biomed Res Int 1:1-9. https://doi.org/10.1155/2018/2543210

Kanehisa M, Goto S (2000) KEGG: kyoto encyclopedia of genes and genomes. Nucleic Acids Res 28(1):27-30

Kanehisa M, Sato Y, Kawashima M, Furumichi M, Tanabe M (2016) KEGG as a reference resource for gene and protein annotation. Nucleic Acids Res 44(D1):D457-D462. https://doi.org/10.1093/nar/gkv1070

Kanehisa M, Furumichi M, Tanabe M, Sato Y, Morishima K (2017) KEGG: new perspectives on genomes, pathways, diseases and drugs. Nucleic Acids Res 45(D1):D353-D361. https://doi.org/10.1093/nar/gkw1092

Kerovuo J, Lauraeus M, Nurminen P, Kalkkinen N, Apajalahti J (1998) Isolation, characterization, molecular gene cloning, and sequencing of a novel phytase from Bacillus subtilis. Appl Environ Microbiol 64(6):2079

Lesuisse E, Schanck K, Colson C (1993) Purification and preliminary characterization of the extracellular lipase of Bacillus subtilis 168, an extremely basic pH-tolerant enzyme. Eur J Biochem 216(1):155-160. https://doi. org/10.1111/j.1432-1033.1993.tb18127.x 
Li B, Lu F, Nan H, Liu Y (2012a) Isolation and structural characterisation of okara polysaccharides. Molecules 17(1):753-761. https://doi.org/10.3390/molec ules17010753

Li B, Qiao M, Lu F (2012b) Composition, nutrition, and utilization of okara (soybean residue). Food Rev Int 28(3):231-252. https://doi.org/10.1080/87559 129.2011.595023

Lopez-Huertas E (2010) Health effects of oleic acid and long chain omega-3 fatty acids (EPA and DHA) enriched milks. A review of intervention studies. Pharmacol Res 61(3):200-207. https://doi.org/10.1016/j. phrs.2009.10.007

Ma J, Zhang Z, Wang B, Kong X, Wang Y, Cao S, Feng Y (2006) Overexpression and characterization of a lipase from Bacillus subtilis. Protein Expr Purif 45(1):22-29. https://doi.org/10.1016/j.pep.2005.06.004

Mienda B, Idi A (2011) Microbiological features of solid state fermentation and its applications. An overview. Res J Biotechnol 2(6):21-26

Nagao K, Yanagita T (2005) Conjugated fatty acids in food and their health benefits. J Biosci Bioeng 100(2):152-157. https://doi.org/10.1263/ jbb.100.152

Ogata H, Goto S, Sato K, Fujibuchi W, Bono H, Kanehisa M (1999) KEGG: Kyoto Encyclopedia of Genes and Genomes. Nucleic Acids Res 27(1):29-34. https://doi.org/10.1093/nar/27.1.29

O'Toole DK (1999) Characteristics and use of okara, the soybean residue from soy milk production a review. J Agric Food Chem 47(2):363-371. https:// doi.org/10.1021/jf9807541

Pandey A, Szakacs G, Soccol C, Rodriguez-Leon JA, Thomaz-Soccol V (2001) Production, purification and properties of microbial phytase. Bioresour Technol 77(3):203-214. https://doi.org/10.1016/S0960-8524(00)00139-5

Paredes-Lopez O, Harry Gl (1989) Changes in selected chemical and antinutritional components during tempeh preparation using fresh and hardened common beans. J Food Sci 54(4):968-970. https://doi. org/10.1111/j.1365-2621.1989.tb07923.x

Rodriguez-Couto S, Sanromán M (2006) Application of solid-state fermentation to food industry-a review. J Food Eng 76(3):291-302. https://doi. org/10.1016/j.jfoodeng.2005.05.022

Ruth MR, Field CJ (2013) The immune modifying effects of amino acids on gut-associated lymphoid tissue. J Anim Sci Biotechnol 4(1):27. https://doi. org/10.1186/2049-1891-4-27

Saif M, Fumio Hashinaga M (2005) Antibacterial and antioxidant activities of banana (Musa, AAA cv. Cavendish) fruits peel. Am J Biochem Biotechnol 1(3):125-131. https://doi.org/10.3844/ajbbsp.2005.125.131

Sánchez M, Prim N, Rández-Gil F, Pastor FIJ, Diaz P (2002) Engineering of baker's yeasts, E. coli and Bacillus hosts for the production of Bacillus subtilis
Lipase A. Biotechnol Bioeng 78(3):339-345. https://doi.org/10.1002/ bit.10201

Schlemmer U, Frolich W, Prieto RM, Grases F (2009) Phytate in foods and significance for humans: food sources, intake, processing, bioavailability, protective role and analysis. Mol Nutr Food Res 53(Suppl 2):S330-S375. https://doi.org/10.1002/mnfr.200900099

Seong Cl, Gyu KY, Kyun JJ, Bae HJ (2015) Soybean waste (okara) as a valorization biomass for the bioethanol production. Energy 93:1742-1747. https ://doi.org/10.1016/j.energy.2015.09.093

Singh KD, Schmalisch MH, Stülke J, Görke B (2008) Carbon catabolite repression in Bacillus subtilis: quantitative analysis of repression exerted by different carbon sources. J Bacteriol 190(21):7275-7284. https://doi. org/10.1128/JB.00848-08

Tian Y, Fan Y, Liu J, Xiangying Z, Chen W (2015) Effect of nitrogen, carbon sources and agitation speed on acetoin production of Bacillus subtilis SF4-3. Electron J Biotechnol 19:41-49. https://doi.org/10.1016/j. ejbt.2015.11.005

Warriner K, Waites WM (1999) Enhanced sporulation in Bacillus subtilis grown on medium containing glucose: ribose. Lett Appl Microbiol 29(2):97-102. https://doi.org/10.1046/j.1365-2672.1999.00593.x

Xia J, Wishart DS (2011) Web-based inference of biological patterns, functions and pathways from metabolomic data using MetaboAnalyst. Nat Protoc 6(6):743-760. https://doi.org/10.1038/nprot.2011.319

Xia J, Wishart DS (2016) Using MetaboAnalyst 3.0 for comprehensive metabolomics data analysis. Curr Protoc Bioinform 55:14

Xia J, Mandal R, Sinelnikov I, Broadhurst D, Wishart DS (2012) MetaboAnalyst 2.0-A comprehensive server for metabolomic data analysis. Nucleic Acids Res 40:W127-W133. https://doi.org/10.1093/nar/gks374

Yang JK, Shih IL, Tzeng YM, Wang S-L (2000) Production and purification of protease from a Bacillus subtilis that can deproteinize crustacean wastes. Enzyme Microb Technol 26(5-6):406-413. https://doi.org/10.1016/S0141 -0229(99)00164-7

Zhu YP, Fan JF, Cheng YQ, Li LT (2008) Improvement of the antioxidant activity of Chinese traditional fermented okara (Meitauza) using Bacillus subtilis B2. Food Control 19(7):654-661. https://doi.org/10.1016/j.foodc ont.2007.07.009

Zong G, Li Y, Wanders AJ, Alssema M, Zock PL, Willett WC, Hu FB, Sun Q (2016) Intake of individual saturated fatty acids and risk of coronary heart disease in US men and women: two prospective longitudinal cohort studies. BMJ 355:i5796. https://doi.org/10.1136/bmj.i5796

\section{Submit your manuscript to a SpringerOpen ${ }^{\odot}$ journal and benefit from:}

- Convenient online submission

- Rigorous peer review

- Open access: articles freely available online

- High visibility within the field

- Retaining the copyright to your article

Submit your next manuscript at $\boldsymbol{\nabla}$ springeropen.com 